\title{
Divisors on graphs, Connected flags, and Syzygies
}

\author{
Fatemeh Mohammadi ${ }^{1} \llbracket$ and Farbod Shokrieh ${ }^{2} \rrbracket$ \\ ${ }^{1}$ Fachbereich Mathematik und Informatik, Philipps-Universität, Marburg, Germany \\ ${ }^{2}$ Georgia Institute of Technology, Atlanta, Georgia, USA
}

\begin{abstract}
We study the binomial and monomial ideals arising from linear equivalence of divisors on graphs from the point of view of Gröbner theory. We give an explicit description of a minimal Gröbner basis for each higher syzygy module. In each case the given minimal Gröbner basis is also a minimal generating set. The Betti numbers of $I_{G}$ and its initial ideal (with respect to a natural term order) coincide and they correspond to the number of "connected flags" in $G$. Moreover, the Betti numbers are independent of the characteristic of the base field.

Résumé. Nous étudions les idéaux monômiaux et binomiaux résultant de l'équivalence linéaire de diviseurs sur les graphes du point de vue de la théorie de Gröbner. Nous donnons une description explicite d'une base de Gröbner minimale pour chaque module engendré par une syzygie d'ordre supérieur. Dans chaque cas, cette base de Gröbner minimale est aussi une ensemble generateur minimal. Les nombres de Betti de $I_{G}$ et son idéal initial coïncident et correspondent au nombre de «drapeaux connexes $\gg$ de $G$. En particulier, les nombres de Betti sont indépendants de la caractéristique du corps de référence.
\end{abstract}

Keywords: Graph, divisors, chip-firing, Gröbner bases, Betti numbers, connected flags.

\section{Introduction}

The theory of divisors on finite graphs can be viewed as a discrete version of the analogous theory on Riemann surfaces. This notion arises in different fields of research including the study of "abelian sandpiles" ([Dha90, Gab93]), the study of component groups of Néron models of Jacobians of algebraic curves ([Ray70, Lor89]), and the theory of chip-firing games on graphs ([Big97]]). Riemann-Roch theory for finite graphs (and generalizations to tropical curves) is developed in this setting ([BN07, GK08, MZ08]).

We are interested in the linear equivalence of divisors on graphs from the point of view of commutative algebra. Associated to every graph $G$ there is a canonical binomial ideal $I_{G}$ which encodes the linear equivalences of divisors on $G$. Let $R$ denote the polynomial ring with one variable associated to each vertex. For any two effective divisors $D_{1} \sim D_{2}$ there is a binomial $\mathbf{x}^{D_{1}}-\mathrm{x}^{D_{2}}$. The ideal $I_{G} \subset R$ is generated by all such binomials. Two effective divisors are linearly equivalent if and only if their associated monomials are equal in $R / I_{G}$. This ideal is already implicitly defined in Dhar's seminal statistical physics paper [Dha90]; $R / I_{G}$ is the "operator algebra" defined there. To our knowledge, this ideal (more precisely, an

\footnotetext{
${ }^{\dagger}$ Supported by the Mathematical Sciences Research Institute and the Alexander von Humboldt Foundation.

${ }^{\ddagger}$ Partially supported by NSF grants DMS-0901487 
affine piece of it) was first introduced in [CRS02] to address computational questions in chip-firing dynamics using Gröbner basis. From a purely computational point of view there are now much more efficient methods available (see, e.g., [BS13] and references therein). However this ideal seems to encode a lot of interesting information about $G$ and its linear systems. Some of the algebraic properties of $I_{G}$ (and its generalization for directed graphs) are studied in [PPW11]. In [MS13a], Manjunath and Sturmfels relate Riemann-Roch theory for finite graphs to Alexander duality in commutative algebra using this ideal.

In this paper we study the syzygies and free resolutions of the ideals $I_{G}$ and $\operatorname{in}\left(I_{G}\right)$ from the point of view of Gröbner theory. Here in $\left(I_{G}\right)$ denotes the initial ideal with respect to a natural term order which is defined after distinguishing a vertex $q$ (see Definition 2.1). When $G$ is a complete graph, the syzygies and Betti numbers of the ideal in $\left(I_{G}\right)$ are studied by Postnikov and Shapiro in [PS04]. Again for complete graphs, Manjunath and Sturmfels in [MS13a] study the ideal $I_{G}$ and show that the Betti numbers coincide with the Betti numbers of in $\left(I_{G}\right)$. Finding minimal free resolutions for a general graph $G$ was stated as an open problem in both [PS04] and [MS13a] (also in [PPW11], where a conjecture is formulated). It was not even known whether the Betti numbers for a general graph depend on the characteristic of the base field or not.

We construct free resolutions for both $\operatorname{in}\left(I_{G}\right)$ and $I_{G}$ for a general graph $G$. Indeed we describe, combinatorially, the minimal Gröbner bases for all higher syzygy modules of $I_{G}$ and $\operatorname{in}\left(I_{G}\right)$. In each case the minimal Gröbner basis is also a minimal generating set and the given resolution is minimal. In particular the Betti numbers of in $\left(I_{G}\right)$ and $I_{G}$ coincide. This gives a positive answer to [CHT06, Question 1.1] for ideal $I_{G}$. For a complete graph the minimal free resolution for $\operatorname{in}\left(I_{G}\right)$ is nicely structured by a Scarf complex. The resolution for $I_{G}$ when $G$ a tree is given by a Koszul complex since $I_{G}$ is a complete intersection. A more conceptual and geometric proof for a general graph $G$ will be given in [MS13b].

The description of the generating sets and the Betti numbers is in terms of the "connected flags" of $G$. Fix a vertex $q \in V(G)$ and an integer $k$. A connected $k$-flag of $G$ (based at $q$ ) is a strictly increasing sequence $U_{1} \subsetneq U_{2} \subsetneq \cdots \subsetneq U_{k}=V(G)$ such that $q \in U_{1}$ and all induced subgraphs on vertex sets $U_{i}$ and $U_{i+1} \backslash U_{i}$ are connected. Associated to any connected $k$-flag one can assign a "partial orientation" on $G$ (Definition 3.3). Two connected $k$-flags are considered equivalent if the associated partially oriented graphs coincide. The Betti numbers correspond to the numbers of the connected flags up to this equivalence. We give a bijective map between the connected flags of $G$ and the minimal Gröbner bases for higher syzygy modules of $I_{G}$ and in $\left(I_{G}\right)$. For a complete graph all flags are connected and all distinct flags are inequivalent. So in this case the Betti numbers are simply the face numbers of the order complex of the poset of those subsets of $V(G)$ that contain $q$ (ordered by inclusion). These numbers can be described using classical Stirling numbers (see Example 4.6. Hence our results directly generalize the analogous results in [PS04] and [MS13a]. Analogous results with different methods were obtained independently in [MSW12] and in [DS12].

The paper is structured as follows. In $\S 2$ we fix our notation and provide the necessary background from the theory of divisors on graphs. We also define the ideal $I_{G}$ and the natural $\operatorname{Pic}(G)$-grading and a term order $<$ on the polynomial ring relevant to our setting. In $\S 2.2$ we quickly recall some basic notions from commutative algebra. Our main goal is to fix our notation for Schreyer's algorithm for computing higher syzygies, which is slightly different from what appears in the existing literature but is more convenient for our application. In $\S 3$ we define connected flags and their equivalence relation. In $\S 4$ we study the free resolution and higher syzygies of our ideals from the point of view of Gröbner theory, and as a corollary 
we give our description of the graded Betti numbers. In $\S 5$ we describe some connections with the theory of reduced divisors. We refer to [MS12] for proofs and more details.

\section{Definitions and background}

\subsection{Graphs and divisors}

Throughout this paper, a graph means a finite, connected, unweighted multigraph with no loops. As usual, the set of vertices and edges of a graph $G$ are denoted by $V(G)$ and $E(G)$. We set $n=|V(G)|$. A pointed graph $(G, q)$ is a graph together with a choice of a distinguished vertex $q \in V(G)$.

Let $\operatorname{Div}(G)$ be the free abelian group generated by $V(G)$. An element of $\operatorname{Div}(G)$ is written as $\sum_{v \in V(G)} a_{v}(v)$ and is called a divisor on $G$. The coefficient $a_{v}$ in $D$ is also denoted by $D(v)$. A divisor $D$ is called effective if $D(v) \geq 0$ for all $v \in V(G)$. The set of effective divisors is denoted by $\operatorname{Div}_{+}(G)$. We write $D \leq E$ if $E-D \in \operatorname{Div}_{+}(G)$. For $D \in \operatorname{Div}(G)$, let $\operatorname{deg}(D)=\sum_{v \in V(G)} D(v)$. For $D_{1}, D_{2} \in \operatorname{Div}(G)$, the divisor $E=\max \left(D_{1}, D_{2}\right)$ is defined by $E(v)=\max \left(D_{1}(v), D_{2}(v)\right)$ for $v \in V(G)$.

We denote by $\mathcal{M}(G)$ the group of integer-valued functions on the vertices. For $A \subseteq V(G), \chi_{A} \in$ $\mathcal{M}(G)$ denotes the $\{0,1\}$-valued characteristic function of $A$. The Laplacian operator $\Delta: \mathcal{M}(G) \rightarrow$ $\operatorname{Div}(G)$ is defined by

$$
\Delta(f)=\sum_{v \in V(G)} \sum_{\{v, w\} \in E(G)}(f(v)-f(w))(v) .
$$

The group of principal divisors is defined as the image of the Laplacian operator and is denoted by $\operatorname{Prin}(G)$. It is easy to check that $\operatorname{Prin}(G) \subseteq \operatorname{Div}^{0}(G)$ where $\operatorname{Div}^{0}(G)$ denotes the set consisting of divisors of degree zero. The quotient $\operatorname{Pic}^{0}(G)=\operatorname{Div}^{0}(G) / \operatorname{Prin}(G)$ is a finite group whose cardinality is the number of spanning trees of $G$ (see, e.g., [BS13] and references therein). The full Picard group of $G$ is defined as

$$
\operatorname{Pic}(G)=\operatorname{Div}(G) / \operatorname{Prin}(G)
$$

which is isomorphic to $\mathbb{Z} \oplus \operatorname{Pic}^{0}(G)$. Since principal divisors have degree zero, the map $\operatorname{deg}: \operatorname{Div}(G) \rightarrow$ $\mathbb{Z}$ descends to a well-defined map $\operatorname{deg}: \operatorname{Pic}(G) \rightarrow \mathbb{Z}$. Two divisors $D_{1}$ and $D_{2}$ are called linearly equivalent if they become equal in $\operatorname{Pic}(G)$. In this case we write $D_{1} \sim D_{2}$. The linear system $|D|$ of $D$ is defined as the set of effective divisors that are linearly equivalent to $D$.

To an ordered pair of disjoint subsets $A, B \subseteq V(G)$ we assign an effective divisor

$$
D(A, B)=\sum_{v \in A}|\{w \in B:\{v, w\} \in E(G)\}|(v) .
$$

In other words, the support of $D(A, B)$ is a subset of $A$ and for $v \in A$ the coefficient of $(v)$ in $D(A, B)$ is the number of edges between $v$ and $B$.

Let $K$ be a field and let $R=K[\mathbf{x}]$ be the polynomial ring in the $n$ variables $\left\{x_{v}: v \in V(G)\right\}$. Any effective divisor $D$ gives rise to a monomial

$$
\mathbf{x}^{D}:=\prod_{v \in V(G)} x_{v}^{D(v)} .
$$


Associated to every graph $G$ there is a canonical ideal which encodes the linear equivalences of divisors on $G$. Our main object study is the ideal

$$
I_{G}:=\left\langle\mathbf{x}^{D_{1}}-\mathbf{x}^{D_{2}}: D_{1} \sim D_{2} \text { both effective divisors }\right\rangle
$$

which was introduced in [CRS02].

Once we fix a vertex $q$, there is a natural monomial order that gives rise to a particularly nice Gröbner basis for $I_{G}$. This term order was first introduced in [CRS02]. Fix a pointed graph $(G, q)$. Consider a total ordering of the set of variables $\left\{x_{v}: v \in V(G)\right\}$ compatible with the distances of vertices from $q$ in $G$ :

$$
\operatorname{dist}(w, q)<\operatorname{dist}(v, q) \Longrightarrow x_{w}<x_{v} .
$$

Here, the distance between two vertices in a graph is the number of edges in a shortest path connecting them. The above ordering can be thought of an ordering on vertices induced by running the breadth-first search algorithm starting at the root vertex $q$.

Definition 2.1 We denote by $<$ the degree reverse lexicographic ordering on $R=K[\mathbf{x}]$ induced by the total ordering on the variables given in (1).

Throughout this paper in $\left(I_{G}\right)$ denotes the initial ideal of $I_{G}$ with respect to this term order. Note that $\operatorname{in}\left(I_{G}\right)$ is denoted by $M_{G}$ in [PS04].

\subsection{Syzygies and Betti numbers}

In this subsection we quickly recall some basic notions from commutative algebra in order to fix our notation. We refer to standard books (e.g. [Eis95, GP08]) for more details.

Let $K$ be any field and let $R=K[\mathbf{x}]$ be the polynomial ring in $n$ variables graded by an abelian group A. The degree map will be denoted by deg. Let $M$ be a graded submodule of a free module and fix a module ordering $<_{0}$ extending the monomial ordering $<$ on $R$. Assume that the finite totally ordered set $(\mathbf{G}, \prec)$ forms a Gröbner basis for $\left(M,<_{0}\right)$ consisting of homogeneous elements. Let $F_{0}$ be the free module generated by $\mathbf{G}$. For $g \in \mathbf{G}$ we let the formal symbol $[g]$ denote the corresponding generator for $F_{0}$; each element of $F_{0}$ can be written as a sum of these formal symbols with coefficients in $R$. There is a natural surjective homomorphism

$$
\varphi_{0}: F_{0} \longrightarrow M
$$

sending $[g]$ to $g$ for each $g \in \mathbf{G}$. Moreover, we enforce this homomorphism to be graded (or homogeneous of degree 0 ) by defining $\operatorname{deg}([g]):=\operatorname{deg}(g)$ for all $g \in \mathbf{G}$.

By definition the syzygy module of $M$ with respect to $\mathbf{G}$, denoted by $\operatorname{syz}(\mathbf{G})$, is the kernel of this map. Let $\operatorname{syz}_{0}(\mathbf{G}):=M$ and $\operatorname{syz}_{1}(\mathbf{G}):=\operatorname{syz}(\mathbf{G})$. For $i>1$ the higher syzygy modules are defined as $\operatorname{syz}_{i}(\mathbf{G}):=\operatorname{syz}\left(\operatorname{syz}_{i-1}(\mathbf{G})\right)$.

We now discuss a method to compute a Gröbner basis for $\operatorname{syz}(\mathbf{G})$. One can "pull back" the module ordering $<_{0}$ along $\varphi_{0}$ to get a compatible module ordering $<_{1}$ on $F_{0}$; for $f, h \in \mathbf{G}$ define

$$
\mathbf{x}^{\beta}[h]<_{1} \mathbf{x}^{\alpha}[f] \Leftrightarrow\left\{\begin{array}{l}
\operatorname{LM}\left(\mathbf{x}^{\beta} h\right)<_{0} \operatorname{LM}\left(\mathbf{x}^{\alpha} f\right) \\
\text { or } \\
\operatorname{LM}\left(\mathbf{x}^{\beta} h\right)=\operatorname{LM}\left(\mathbf{x}^{\alpha} f\right) \wedge f \prec h .
\end{array}\right.
$$


To simplify the notation we assume the leading coefficients of all elements of $\mathbf{G}$ are 1. For a pair of elements $f \prec h$ of $\mathbf{G}$ assume

$$
\operatorname{LM}(f)=\mathbf{x}^{\alpha(f)}[e] \quad \text { and } \quad \operatorname{LM}(h)=\mathbf{x}^{\alpha(h)}[e]
$$

for some $e \in E$. Since $\mathbf{G}$ is a Gröbner basis, setting $\gamma(f, h):=\max (\alpha(f), \alpha(h))$, we have the "standard representation":

$$
\operatorname{spoly}(f, h)=\mathbf{x}^{\gamma(f, h)-\alpha(f)} f-\mathbf{x}^{\gamma(f, h)-\alpha(h)} h=\sum_{g \in \mathbf{G}} a_{g}^{(f, h)} g
$$

for some polynomials $a_{g}^{(f, h)} \in R$. We set

$$
s(f, h)=\mathbf{x}^{\gamma(f, h)-\alpha(f)}[f]-\mathbf{x}^{\gamma(f, h)-\alpha(h)}[h]-\sum_{g \in \mathbf{G}} a_{g}^{(f, h)}[g] \in F_{0} .
$$

Theorem 2.2 (Schreyer [Sch80], [Eis95]) The set

$$
\mathcal{S}(\mathbf{G})=\left\{s(f, h): f, h \in \mathbf{G}, f \prec h, \operatorname{LM}(f)=\mathbf{x}^{\alpha(f)}[e], \operatorname{LM}(h)=\mathbf{x}^{\alpha(h)}[e] \text { for some } e \in E\right\}
$$

forms a homogeneous Gröbner basis for $\left(\operatorname{syz}(\mathbf{G}),<_{1}\right)$.

To read the Betti numbers for $M$ one needs to find a minimal generating set for the syzygy modules. In general the set $\mathcal{S}(\mathbf{G})$ is far from being even a minimal Gröbner basis. However there exist some criterions to find a subset $\mathcal{S}_{\min }(\mathbf{G})$ of $\mathcal{S}(\mathbf{G})$ which forms a minimal Gröbner basis for $\left(\operatorname{syz}(\mathbf{G}),<_{1}\right)$; see, e.g., [MS12, Lemma 3.4]. Moreover, Theorem 2.2 gives rise to Algorithm 1 for computing free resolutions. The following result gives a general sufficient criterion for an ideal to have the same Betti numbers as its initial ideal.

Theorem 2.3 If the constructed resolution by Schreyer's algorithm is a minimal graded free resolution then $\beta_{i, \mathrm{j}}(M)=\beta_{i, \mathrm{j}}(\mathrm{in}(M))$ for all $i \geq 0$ and $\mathrm{j} \in \mathrm{A}$.

\section{Connected flags on graphs}

\subsection{Connected flags, partial orientations, and divisors}

From now on we fix a pointed graph $(G, q)$ and we let $n=|V(G)|$. Consider the poset

$$
\mathfrak{C}(G, q):=\{U \subseteq V(G): q \in U\}
$$

ordered by inclusion. The following special chains of this poset arise naturally in our setting.

Definition 3.1 Fix an integer $1 \leq k \leq n$. A connected $k$-flag of $(G, q)$ is a (strictly increasing) sequence $\mathcal{U}$ of subsets of $V(G)$

$$
U_{1} \subsetneq U_{2} \subsetneq \cdots \subsetneq U_{k}=V(G)
$$

such that $q \in U_{1}$ and, for all $1 \leq i \leq k-1$, both $G\left[U_{i}\right]$ and $G\left[U_{i+1} \backslash U_{i}\right]$ are connected.

The set of all connected $k$-flags of $(G, q)$ will be denoted by $\mathfrak{F}_{k}(G, q)$. 


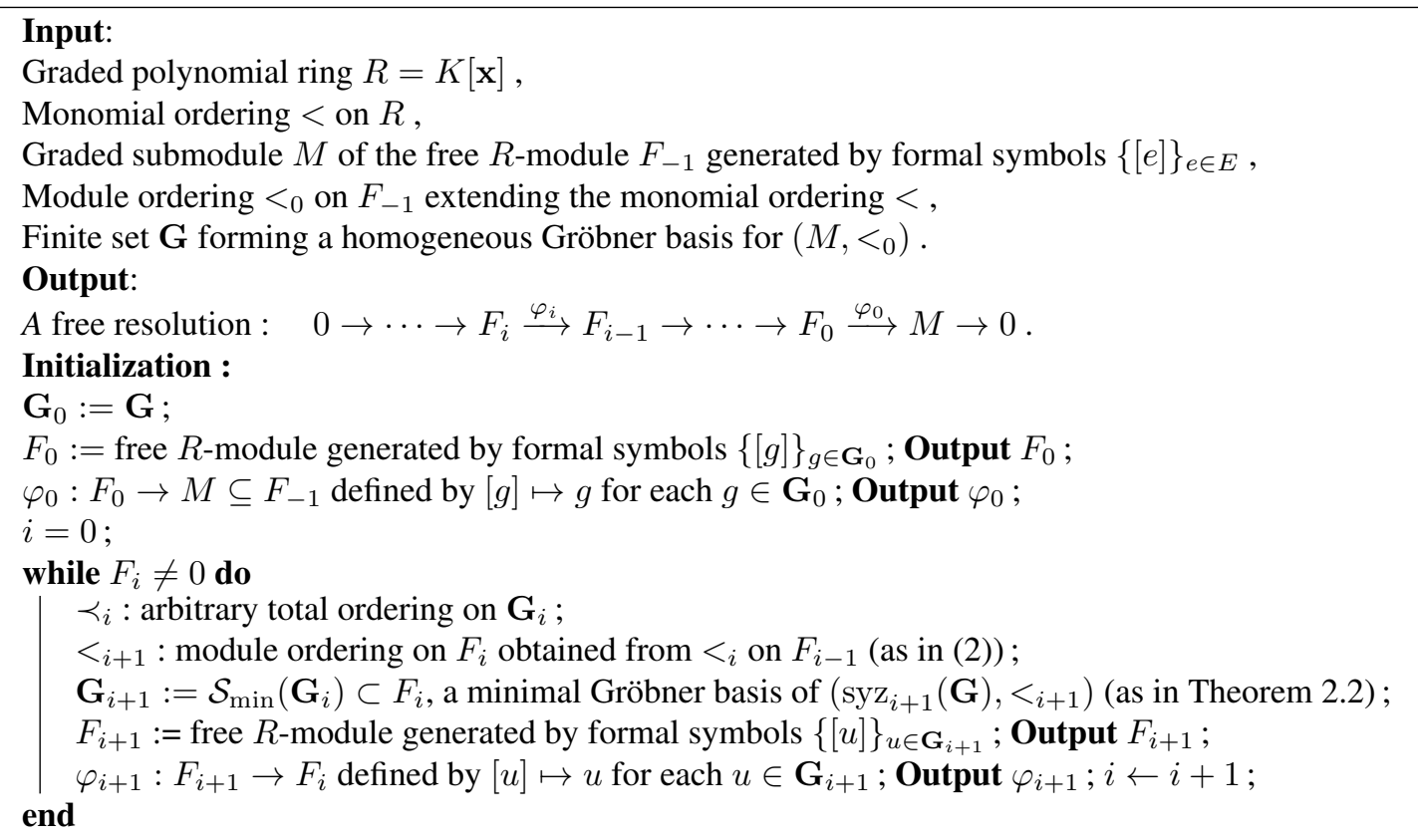

Algorithm 1: Algorithm for computing a free resolution of $M$ (Schreyer's algorithm)

Remark 3.2 For a complete graph, $\mathfrak{F}_{k}(G, q)$ is simply the order complex of $\mathfrak{C}(G, q)$, but in general $\mathfrak{F}_{k}(G, q)$ is not a simplicial complex.

Definition 3.3 Given $\mathcal{U} \in \mathfrak{F}_{k}(G, q)$ we define :

(a) a "partial orientation" of $G$ by orienting edges from $U_{i}$ to $U_{i+1} \backslash U_{i}$ (for all $1 \leq i \leq k-1$ ) and

leaving all other edges unoriented. We denote the resulting partially oriented graph by $G(\mathcal{U})$.

(b) an effective divisor $D(\mathcal{U}) \in \operatorname{Div}(G)$ given by $D(\mathcal{U}):=\sum_{i=1}^{k-1} D\left(U_{i+1} \backslash U_{i}, U_{i}\right)$.

Remark 3.4 It is easy to check that $D(\mathcal{U})=\sum_{v \in V(G)}\left(\operatorname{indeg}_{G(\mathcal{U})}(v)\right)(v)$, where $\operatorname{indeg}_{G(\mathcal{U})}(v)$ denotes the number of oriented edges directed to $v$ in $G(\mathcal{U})$.

\subsection{Total ordering on $\mathfrak{F}_{k}(G, q)$}

We endow each $\mathfrak{F}_{k}(G, q)$ with a total orderings $\prec_{k}$ for all $1 \leq k \leq n$. These total orderings are compatible with each other for different values of $1 \leq k \leq n$.

Let $\preceq$ denote the ordering on $\mathfrak{C}^{\text {op }}(G, q)$ given by reverse inclusion :

$$
U \preceq V \Longleftrightarrow U \supseteq V .
$$

Definition 3.5 We fix, once and for all, a total ordering extending $\preceq$. By a slight abuse of notation, $\preceq$ will be used to denote this total ordering extension. In particular $\prec$ will denote the associated strict total order. 
We consider one of the natural "lexicographic extensions" of $\prec$ to the set of connected $k$-flags.

Definition 3.6 For $\mathcal{U} \neq \mathcal{V}$ in $\mathfrak{F}_{k}(G, q)$ written as

$$
\begin{aligned}
& \mathcal{U}: U_{1} \subsetneq U_{2} \subsetneq \cdots \subsetneq U_{k}=V(G) \\
& \mathcal{V}: V_{1} \subsetneq V_{2} \subsetneq \cdots \subsetneq V_{k}=V(G)
\end{aligned}
$$

we say $\mathcal{U} \prec_{k} \mathcal{V}$ iffor the maximum $1 \leq \ell \leq k-1$ with $U_{\ell} \neq V_{\ell}$ we have $\mathcal{U}_{\ell} \prec \mathcal{V}_{\ell}$.

As usual, we write $\mathcal{U} \preceq_{k} \mathcal{V}$ if and only if $\mathcal{U} \prec_{k} \mathcal{V}$ or $\mathcal{U}=\mathcal{V}$.

Lemma $3.7\left(\mathfrak{F}_{k}(G, q), \preceq_{k}\right)$ is a totally ordered set.

It is easy to find two different connected $k$-flags having identical associated partially oriented graphs. This motivates the following definition.

Definition 3.8 Two $k$-flags $\mathcal{U}, \mathcal{V} \in \mathfrak{F}_{k}(G, q)$ are called equivalent if the associated partially oriented graphs $G(\mathcal{U})$ and $G(\mathcal{V})$ coincide.

Notation 1 The set of all equivalence classes in $\mathfrak{F}_{k}(G, q)$ will be denoted by $\mathfrak{E}_{k}(G, q)$. The set $\mathfrak{S}_{k}(G, q)$ denotes the collection of minimal representatives of the classes in $\mathfrak{E}_{k}(G, q)$ with respect to $\prec_{k}$.

Given an element in $\mathfrak{S}_{k}(G, q)$ there is a canonical way to obtain two related elements in $\mathfrak{S}_{k-1}(G, q)$.

Definition 3.9 Given $\mathcal{U} \in \mathfrak{F}_{k}(G, q)$, the elements $\mathcal{U}^{(1)}, \mathcal{U}^{(2)} \in \mathfrak{F}_{k-1}(G, q)$ are obtained from $\mathcal{U}$ by removing the $1^{\text {st }}$ and $2^{\text {nd }}$ elements in the following appropriate sense. Let

$$
\mathcal{U}: U_{1} \subsetneq U_{2} \subsetneq \cdots \subsetneq V(G)
$$

(a) $\mathcal{U}^{(1)}$ will denote

$$
U_{2} \subsetneq U_{3} \subsetneq U_{4} \subsetneq \cdots \subsetneq V(G)
$$

(b) $\mathcal{U}^{(2)}$ will denote

$$
\begin{cases}U_{1} \subsetneq U_{3} \subsetneq U_{4} \subsetneq \cdots \subsetneq V(G), & \text { if } G\left[U_{3} \backslash U_{1}\right] \text { is connected } \\ \text { or } & \\ \left(U_{1} \cup\left(U_{3} \backslash U_{2}\right)\right) \subsetneq U_{3} \subsetneq U_{4} \subsetneq \cdots \subsetneq V(G), & \text { if } G\left[U_{3} \backslash U_{1}\right] \text { is not connected }\end{cases}
$$

Remark 3.10 [MS12, Section 6.1] Assume that $\mathcal{U} \in \mathfrak{S}_{k}(G, q)$. Let $G / \mathcal{U}$ be the graph obtained from $G$ by contracting the unoriented edges of $G(\mathcal{U})$ and let $\phi: G \rightarrow G / \mathcal{U}$ be the contraction map. More precisely, $G_{/ \mathcal{U}}$ is the graph on the vertices $u_{1}, \ldots, u_{k}$ corresponding to the collection $\left(U_{i} \backslash U_{i-1}\right)_{i=1}^{k}$, i.e. $u_{i}=\phi\left(U_{i} \backslash U_{i-1}\right)$. For any edge between $U_{i} \backslash U_{i-1}$ and $U_{j} \backslash U_{j-1}$ there is an edge between $u_{i}$ and $u_{j}$. The contraction map $\phi: G \rightarrow G_{/ \mathcal{U}}$ induces the maps

(i) $\phi_{*}: \operatorname{Div}(G) \rightarrow \operatorname{Div}\left(G_{/ \mathcal{U}}\right)$ with $\phi_{*}\left(\sum_{v \in V(G)} a_{v}(v)\right)=\sum_{v \in V(G)} a_{v}(\phi(v))$. In particular, a total ordering $u_{1}, \ldots, u_{k}$ of $V\left(G_{/ \mathcal{U}}\right)$ gives a total ordering on the collection of subsets $\left(U_{i} \backslash U_{i-1}\right)_{i=1}^{k}$ of $V(G)$. By Definition 3.3 we get a divisor $D^{\prime}$ on $G / \mathcal{U}$ and a divisor $D$ on $G$, and $\phi_{*}(D)=D^{\prime}$. In other words, such a divisor $D^{\prime}$ has a canonical section.

(ii) $\phi^{*}: \mathfrak{S}_{s}\left(G / \mathcal{U}, u_{1}\right) \rightarrow \mathfrak{S}_{s}(G, q)$ with $\phi^{*}\left(\mathcal{V}^{\prime}\right)=\mathcal{V}$ where $V_{j}=\bigcup_{u_{i} \in V_{j}^{\prime}}\left(U_{i} \backslash U_{i-1}\right)$. 


\section{Minimal free resolution and Betti numbers for $I_{G}$ and $\operatorname{in}\left(I_{G}\right)$}

Let $K$ be a field and let $R=K[\mathbf{x}]$ be the polynomial ring in $n$ variables $\left\{x_{v}: v \in V(G)\right\}$. Recall that $K[\mathbf{x}]$ has a natural A-grading, where $\mathrm{A}=\mathbb{Z}$ or $A=\operatorname{Pic}(G)$ and $I_{G}$ is also A-graded. Let the monomial ordering $<$ on $R$ be as in Definition 2.1.

The following theorem gives a generalization of [CRS02, Theorem 14]. Indeed [CRS02, Theorem 14] can be rephrased as providing a bijection between $\mathfrak{S}_{2}(G, q)$ and $\mathbf{G}(G, q)$.

Theorem 4.1 Fix a pointed graph $(G, q)$ and let $\mathrm{A}=\mathbb{Z}$ or $\mathrm{A}=\operatorname{Pic}(G)$. For each $k \geq 0$ there exists a natural injection

$$
\psi_{k}: \mathfrak{S}_{k+2}(G, q) \hookrightarrow \operatorname{syz}_{k}(\mathbf{G}(G, q))
$$

such that

(i) For some module ordering $<_{k}$, the set $\mathbf{G}_{k}(G, q):=\operatorname{Image}\left(\psi_{k}\right)$ forms a minimal A-homogeneous Gröbner basis of $\left(\operatorname{syz}_{k}(\mathbf{G}(G, q)),<_{k}\right)$,

(ii) For $\mathcal{U} \in \mathfrak{S}_{k+2}(G, q)$ of the form $U_{1} \subsetneq U_{2} \subsetneq \cdots \subsetneq V(G)$ we have

$$
\operatorname{LM}\left(\psi_{k}(\mathcal{U})\right)=\mathbf{x}^{D\left(U_{2} \backslash U_{1}, U_{1}\right)}\left[\psi_{k-1}\left(\mathcal{U}^{(1)}\right)\right],
$$

(iii) The set $\psi_{k}\left(\mathfrak{S}_{k+2}(G, q)\right)$ minimally generates $\operatorname{syz}_{k}(\mathbf{G}(G, q))$.

Sketch of proof : Here we list the key steps of the proof. For a complete proof we refer to [MS12]. For consistency in the notation we define $\operatorname{syz}_{-1}(\mathbf{G}(G, q))=\{0\}$ and the map

$$
\psi_{-1}: \mathfrak{S}_{1}(G, q) \hookrightarrow\{0\}
$$

sends the canonical connected 1-flag $V(G)$ to 0 . The proof is by induction on $k \geq 0$.

Base case. For $k=0$ the result is proved in [CRS02, Theorem 14]. Here $\mathbf{G}_{0}(G, q)=\mathbf{G}(G, q)$ and $<_{0}$ is $<$, and

$$
\begin{gathered}
\psi_{0}: \mathfrak{S}_{2}(G, q) \hookrightarrow \operatorname{syz}_{0}(\mathbf{G}(G, q))=I_{G} \\
\left(U_{1} \subsetneq U_{2}\right) \mapsto\left(\mathbf{x}^{D\left(U_{2} \backslash U_{1}, U_{1}\right)}-\mathbf{x}^{D\left(U_{1}, U_{2} \backslash U_{1}\right)}\right)[0]
\end{gathered}
$$

and $\operatorname{LM}\left(\psi_{k}(\mathcal{U})\right)=\mathbf{x}^{D\left(U_{2} \backslash U_{1}, U_{1}\right)}[0]$.

Induction hypothesis. Now let $k>0$ and assume that there exists a bijection

$$
\psi_{k-1}: \mathfrak{S}_{k+1}(G, q) \rightarrow \mathbf{G}_{k-1}(G, q) \subseteq \operatorname{syz}_{k-1}(\mathbf{G}(G, q))
$$

such that $\mathbf{G}_{k-1}(G, q)$ forms a minimal homogeneous Gröbner basis of $\operatorname{syz}_{k-1}(\mathbf{G}(G, q))$ with respect to $<_{k-1}$ ), and (5) for the leading monomials holds.

Via the bijection $\psi_{k-1}$, the set $\mathbf{G}_{k-1}(G, q)$ inherits a total ordering $\prec_{k-1}^{\prime}$ from the total ordering $\prec_{k+1}$ on $\mathfrak{S}_{k+1}(G, q)$, i.e.

$$
f \prec_{k-1}^{\prime} h \quad \text { in } \quad \mathbf{G}_{k-1}(G, q) \Leftrightarrow \psi_{k-1}^{-1}(f) \prec_{k+1} \psi_{k-1}^{-1}(h) \quad \text { in } \quad \mathfrak{S}_{k+1}(G, q) .
$$

Inductive step. Given $\mathcal{U} \in \mathfrak{S}_{k+2}(G, q)$ let $\mathcal{U}^{(1)}$ and $\mathcal{U}^{(2)}$ be as defined in Definition 3.9 We define

$$
\psi_{k}: \mathfrak{S}_{k+2}(G, q) \rightarrow \operatorname{syz}_{k}(\mathbf{G}(G, q))
$$


Divisors on graphs, Connected flags, and Syzygies

$$
\mathcal{U} \mapsto s\left(\psi_{k-1}\left(\mathcal{U}^{(1)}\right), \psi_{k-1}\left(\mathcal{U}^{(2)}\right)\right) .
$$

In the following $\mathcal{U}, \mathcal{V} \in \mathfrak{S}_{k+2}(G, q)$ are of the form

$$
\begin{aligned}
& U_{1} \subsetneq U_{2} \subsetneq \cdots \subsetneq V(G) \\
& V_{1} \subsetneq V_{2} \subsetneq \cdots \subsetneq V(G) .
\end{aligned}
$$

The result is follows from a series of claims.

Claim 1. $\psi_{k}$ is a well-defined and $\mathbf{G}_{k}(G, q):=\operatorname{Image}\left(\psi_{k}\right)$ consists of homogeneous elements.

Since $\psi_{k-1}\left(\mathcal{U}^{(1)}\right)$ and $\psi_{k-1}\left(\mathcal{U}^{(2)}\right)$ are homogeneous by induction hypothesis, it follows that $s\left(\psi_{k-1}\left(\mathcal{U}^{(1)}\right), \psi_{k-1}\left(\mathcal{U}^{(2)}\right)\right)$ is also homogeneous.

Claim 2. $\operatorname{LM}\left(\psi_{k}(\mathcal{U})\right)=\mathbf{x}^{D\left(U_{2} \backslash U_{1}, U_{1}\right)}\left[\psi_{k-1}\left(\mathcal{U}^{(1)}\right)\right]$.

It suffices to show that $D\left(U_{2} \backslash U_{1}, U_{1}\right)=\max (\alpha, \beta)-\alpha$ where

$$
\operatorname{LM}\left(\psi_{k-1}\left(\mathcal{U}^{(1)}\right)\right)=\mathbf{x}^{\alpha}\left[\psi_{k-2}\left(\mathcal{U}^{(1,1)}\right)\right] \quad, \quad \operatorname{LM}\left(\psi_{k-1}\left(\mathcal{U}^{(2)}\right)\right)=\mathbf{x}^{\beta}\left[\psi_{k-2}\left(\mathcal{U}^{(2,1)}\right)\right] .
$$

Claim 3. $\psi_{k}$ is injective.

If $\mathcal{U}, \mathcal{V} \in \mathfrak{S}_{k+2}(G, q)$ be such that $\psi_{k}(\mathcal{U})=\psi_{k}(\mathcal{V})$ then their leading terms are equal :

$$
\mathbf{x}^{D\left(U_{2} \backslash U_{1}, U_{1}\right)}\left[\psi_{k-1}\left(\mathcal{U}^{(1)}\right)\right]=\mathbf{x}^{D\left(V_{2} \backslash V_{1}, V_{1}\right)}\left[\psi_{k-1}\left(\mathcal{V}^{(1)}\right)\right] .
$$

Therefore $\psi_{k-1}\left(\mathcal{U}^{(1)}\right)=\psi_{k-1}\left(\mathcal{V}^{(1)}\right)$ and $D\left(U_{2} \backslash U_{1}, U_{1}\right)=D\left(V_{2} \backslash V_{1}, V_{1}\right)$. By induction hypothesis $\psi_{k-1}$ is injective which implies $\mathcal{U}^{(1)}=\mathcal{V}^{(1)}$ and $D\left(U_{2} \backslash U_{1}, U_{1}\right)=D\left(V_{2} \backslash V_{1}, V_{1}\right)$. Therefore $U_{1}=V_{1}$ and $\mathcal{U}=\mathcal{V}$.

The following claim (proved in [MS12]) will finish the inductive step.

Claim 4. Image $\left(\psi_{k}\right)$ forms a minimal homogeneous Gröbner basis of $\operatorname{syz}_{k}(\mathbf{G}(G, q))$ with respect to $<_{k}$ obtained from $<_{k-1}$ according to 2$]$.

These all together show that Image $\left(\psi_{k}\right) \subseteq \mathcal{S}\left(\mathbf{G}_{k-1}(G, q)\right)$. In order to show the reverse inclusion by Theorem 2.2 it remains to show that

(I) $0 \notin$ Image $\left(\psi_{k}\right)$.

(II) For any element $s(f, h) \in \mathcal{S}\left(\mathbf{G}_{k-1}(G, q)\right)$ there exists an element $g \in \operatorname{Image}\left(\psi_{k}\right)$ such that $\operatorname{LM}(g) \mid \operatorname{LM}(s(f, h))$.

(III) For any two elements $g, g^{\prime} \in \operatorname{Image}\left(\psi_{k}\right)$, if $\operatorname{LM}(g) \mid \operatorname{LM}\left(g^{\prime}\right)$ then $g=g^{\prime}$.

Claim 5. For $\mathcal{U} \in \mathfrak{S}_{k+2}(G, q)$ we have $\psi_{k}(\mathcal{U})=\sum_{\mathcal{W} \in \mathfrak{S}_{k+1}(G, q)} c(\mathcal{U}, \mathcal{W}) \mathbf{x}^{\theta(\mathcal{U}, \mathcal{W})}\left[\psi_{k-1}(\mathcal{W})\right]$ where $c(\mathcal{U}, \mathcal{W}) \in\{-1,0,1\}$ and $\theta(\mathcal{U}, \mathcal{W})=D\left(U_{i} \backslash U_{i-1}, U_{j} \backslash U_{j-1}\right)$ if $\mathcal{W}$ differs from $\mathcal{U}$ by merging $U_{i} \backslash U_{i-1}$ and $U_{j} \backslash U_{j-1}$ for some $i, j$.

Note that this proves (III) which is equivalent to the minimality of the resolution.

From Theorem 4.1 we obtain the following important corollaries. 
Corollary 4.2 The Betti numbers of the ideals $I_{G}$ and $\operatorname{in}\left(I_{G}\right)$ are independent of the characteristic of the base field $K$.

Corollary 4.3 For all $i \geq 0, \beta_{i}\left(R / I_{G}\right)=\beta_{i}\left(R / \operatorname{in}\left(I_{G}\right)\right)=\left|\mathfrak{S}_{i+1}(G, q)\right|=\left|\mathfrak{E}_{i+1}(G, q)\right|$.

Let $\mathrm{A}=\mathbb{Z}$ or A $=\operatorname{Pic}(G)$. Recall that $I_{G}$ and in $\left(I_{G}\right)$ are graded (or homogeneous) with respect to the $\mathbb{Z}$ and $\operatorname{Pic}(G)$ gradings. One can also read the graded Betti numbers from Theorem 4.1 .

Corollary 4.4 For all $i \geq 0$ and $\mathrm{j} \in \mathrm{A}$ we have $\beta_{i, \mathrm{j}}=\left|\mathfrak{S}_{i+1, \mathrm{j}}(G, q)\right|$ where $\mathfrak{S}_{k, \mathrm{j}}(G, q)=\{\mathcal{U} \in$ $\left.\mathfrak{S}_{k}(G, q): \operatorname{deg}_{\mathrm{A}}\left(\mathbf{x}^{D(\mathcal{U})}\right)=\mathrm{j}\right\}$.

We conclude this section with some examples.

Example 4.5 It follows from above descriptions that $\beta_{n-1}\left(R / I_{G}\right)=\beta_{n-1, m}\left(R / I_{G}\right)$ is equal to the number of acyclic orientations of $G$ with unique source.

Example 4.6 Let $G$ be the complete graph $K_{n}$ on $n$ vertices. Then $\beta_{k-1}\left(R / I_{G}\right)=\left|\mathfrak{S}_{k}(G, q)\right|=(k-$ $1) ! S(n, k)$ where $S(n, k)$ denotes the Stirling number of the second kind (i.e. the number of ways to partition a set of $n$ elements into $k$ nonempty subsets).

Example 4.7 Let $G$ be a tree on $n$ vertices. Then $\beta_{k-1}\left(R / I_{G}\right)=\left|\mathfrak{S}_{k}(G, q)\right|=\left(\begin{array}{c}n-1 \\ k-1\end{array}\right)$.

Example 4.8 For the cycle $C_{n}$ on $n$ vertices and $k \geq 2$ we have $\beta_{k-1}\left(R / I_{C_{n}}\right)=\left|\mathfrak{S}_{k}\left(C_{n}, q\right)\right|=(k-$ 1) $\times\left(\begin{array}{l}n \\ k\end{array}\right)$.

\section{Relation to maximal reduced divisors}

Recall the definition of reduced divisors.

Definition 5.1 Let $\left(\Gamma, v_{0}\right)$ be a pointed graph. A divisor $D \in \operatorname{Div}(\Gamma)$ is called $v_{0}$-reduced if it satisfies the following two conditions :

(i) $D(v) \geq 0$ for all $v \in V(\Gamma) \backslash\left\{v_{0}\right\}$.

(ii) For every non-empty subset $A \subseteq V(\Gamma) \backslash\left\{v_{0}\right\}$, there exists a vertex $v \in A$ such that $D(v)<$ $\operatorname{outdeg}_{A}(v)$.

These divisors arise precisely from the normal forms with respect to the Gröbner basis given in Theorem 4.1. There is a well-known algorithm due to Dhar for checking whether a given divisor is reduced (see, e.g., [BS13] and references therein).

Lemma 5.2 For $\mathcal{U} \in \mathfrak{S}_{k}(G, q)$, $\phi_{*}(D(\mathcal{U}))=E+\mathbf{1}$, where $E$ is a maximal $\left(\phi\left(U_{1}\right)\right)$-reduced divisor and 1 is the all-one divisor.

Since different acyclic orientations with unique source at $q^{\prime}$ give rise to inequivalent $q^{\prime}$-reduced divisors we deduce that if $\mathcal{U}, \mathcal{V} \in \mathfrak{S}_{k}(G, q)$ and the graphs $G_{/ \mathcal{U}}$ and $G_{/ \mathcal{V}}$ coincide, then $\phi_{*}(D(\mathcal{U}))-\mathbf{1}$ and $\phi_{*}(D(\mathcal{V}))-\mathbf{1}$ are two inequivalent maximal reduced divisors. These observations lead to the following formula for Betti numbers which was conjectured in [PPW11] for $I_{G}$ :

$$
\begin{aligned}
\beta_{i} & =\sum_{G / \mathcal{U}} \mid\left\{D: D \text { is a maximal } v_{0} \text {-reduced divisors on } G_{/ \mathcal{U}}\right\} \mid \\
& =\sum_{G / \mathcal{U}} \mid\left\{\text { acyclic orientations of } G_{/ \mathcal{U}} \text { with unique source at } v_{0}\right\} \mid
\end{aligned}
$$


where the sum is over all distinct contracted graphs $G_{/ \mathcal{U}}$ as $\mathcal{U}$ varies in $\mathfrak{S}_{i+1}(G, q)$, and $v_{0}$ is an arbitrary vertex of $G_{/ \mathcal{U}}$.

Here is another connection with reduced divisors. Hochster's formula for computing the Betti numbers topologically (see, e.g., MS05, Theorem 9.2]), when applied to $I_{G}$ and the "nice" grading by $\operatorname{Pic}(G)$, says that for each $\mathrm{j} \in \operatorname{Pic}(G)$ the graded Betti number $\beta_{i, \mathrm{j}}\left(R / I_{G}\right)$ is the dimension of the $i^{\text {th }}$ reduced homology of the simplicial complex $\Delta_{\mathrm{j}}=\left\{\operatorname{supp}(E): 0 \leq E \leq D^{\prime} \in|\mathrm{j}|\right\}$ where $|\mathrm{j}|$ denotes the linear system of $\mathrm{j} \in \operatorname{Pic}(G)$.

\section{Remark 5.3}

(i) For $\mathrm{j} \in \operatorname{Pic}(G)$, we have $\beta_{n-1, \mathrm{j}}\left(R / I_{G}\right)=1$ if and only if $\mathrm{j} \sim E+\mathbf{1}$ where $E$ is a maximal $q$-reduced divisor.

(ii) One can use Corollary 4.3 to read all dimensions of the reduced homologies of $\Delta_{\mathrm{j}}$. Although we now know all the Betti numbers, giving an explicit bijection between connected flags and the bases of the reduced homologies of $\Delta_{\mathrm{j}}$ is an intriguing problem. In a recent work, Horia Mania in [Man12] studies the number of connected components of $\Delta_{\mathrm{j}}$.

\section{Acknowledgements}

The first author is grateful to Hélène Barcelo and Volkmar Welker for numerous comments and many helpful conversations. Part of this work was done while the second author was visiting UC Berkeley, which he would like to thank for the hospitality.

\section{Références}

[Big97] N. Biggs. Algebraic potential theory on graphs. Bull. London Math. Soc., 29(6) :641-682, 1997.

[BN07] M. Baker and S. Norine. Riemann-Roch and Abel-Jacobi theory on a finite graph. Adv. Math., 215(2) :766-788, 2007.

[BS13] M. Baker and F. Shokrieh. Chip-firing games, potential theory on graphs, and spanning trees. Journal of Combinatorial Theory, Series A, 120(1) :164 - 182, 2013.

[CHT06] A. Conca, S. Hoşten, and R. R. Thomas. Nice initial complexes of some classical ideals. In Algebraic and geometric combinatorics, volume 423 of Contemp. Math., pages 11-42. Amer. Math. Soc., Providence, RI, 2006.

[CRS02] R. Cori, D. Rossin, and B. Salvy. Polynomial ideals for sandpiles and their Gröbner bases. Theoret. Comput. Sci., 276(1-2) :1-15, 2002.

[Dha90] D. Dhar. Self-organized critical state of sandpile automaton models. Phys. Rev. Lett., 64(14) :1613-1616, Apr 1990.

[DS12] A. Dochtermann and R. Sanyal. Laplacian ideals, arrangements, and resolutions. Preprint available at arXiv:1212.624 4, 2012.

[Eis95] D. Eisenbud. Commutative algebra, with a view toward algebraic geometry, volume 150 of Graduate Texts in Mathematics. Springer-Verlag, New York, 1995.

[Gab93] A. Gabrielov. Abelian avalanches and Tutte polynomials. Phys. A, 195(1-2) :253-274, 1993. 
[GK08] A. Gathmann and M. Kerber. A Riemann-Roch theorem in tropical geometry. Math. Z., 259(1) :217-230, 2008.

[GP08] G. Greuel and G. Pfister. A Singular introduction to commutative algebra. Springer, Berlin, extended edition, 2008.

[Lor89] D. Lorenzini. Arithmetical graphs. Math. Ann., 285(3) :481-501, 1989.

[Man12] H. Mania. Wilmes' conjecture and boundary divisors. Preprint, 2012.

[MS05] E. Miller and B. Sturmfels. Combinatorial commutative algebra, volume 227 of Graduate Texts in Mathematics. Springer-Verlag, New York, 2005.

[MS12] F. Mohammadi and F. Shokrieh. Divisors on graphs, connected flags, and syzygies. Preprint available at arXiv:1210.6622, 2012.

[MS13a] M. Manjunath and B. Sturmfels. Monomials, binomials and Riemann-Roch. Journal of Algebraic Combinatorics, pages 1-20, 2013. to appear.

[MS13b] F. Mohammadi and F. Shokrieh. Divisors on graphs and cellular resolutions. In preparation, 2013.

[MSW12] M. Manjunath, F. Schreyer, and J. Wilmes. Minimal free resolutions of the $g$-parking function ideal and the toppling ideal. Preprint available at arXiv:1210.7569v2, 2012.

[MZ08] G. Mikhalkin and I. Zharkov. Tropical curves, their Jacobians and theta functions. In Curves and abelian varieties, volume 465 of Contemp. Math., pages 203-230. Amer. Math. Soc., Providence, RI, 2008.

[PPW11] D. Perkinson, J. Perlman, and J. Wilmes. Primer for the algebraic geometry of sandpiles. 2011. Preprint available at arXiv:1112.6163.

[PS04] A. Postnikov and B. Shapiro. Trees, parking functions, syzygies, and deformations of monomial ideals. Trans. Amer. Math. Soc., 356(8) :3109-3142 (electronic), 2004.

[Ray70] M. Raynaud. Spécialisation du foncteur de Picard. Inst. Hautes Études Sci. Publ. Math., (38) :27-76, 1970.

[Sch80] F. Schreyer. Die Berechnung von Syzygien mit dem verallgemeinerten Weierstrass'schen Divisionssatz. Diplom Thesis, University of Hamburg, Germany., 1980. 